\title{
A context aware ar chitecture to support people with partial visual impairments
}

\author{
João Fernandes ${ }^{\mathrm{a}}$, João Laranjeir $\mathrm{a}^{\mathrm{c}}$, Paulo Novais ${ }^{\mathrm{a}}$, Gor eti Marreiros ${ }^{\mathrm{b}, \mathrm{d}}$, \\ José Neves ${ }^{\mathrm{a}}$ \\ aUniversity of Minho \\ b Institute of Engineering - Polytechnic of Porto \\ ${ }^{\mathrm{C} C C T C}$ - Computer Science and Technology Center \\ ${ }^{d}$ GECAD - Knowledge Engineering and Decision Support Group \\ pg20686@alunos.uminho.pt; jopcl@isep.ipp.pt; pjon@di.uminho.pt; mgt@isep.ipp.pt; \\ jneves@di.uminho.pt
}

\begin{abstract}
Nowadays there are several systems that help people with disabilities on their quotidian tasks. The visual impairment is a problem that affects several people in their tasks and movements. In this work we propose an architecture capable of processing information from the environment and suggesting actions to the user with visual impairments, to avoid a possible obstacle. This architecture intends to improve the support given to the user in their daily movements. The idea is to use speculative computation to predict the users' intentions and even to justify the reactive or proactive users' behaviors.
\end{abstract}

Keywords: decision support system, ambient intelligence, speculative computation

\section{Intr oduction}

Nowadays there are many systems that assist/help the users on taking decisions in order to make their life more comfortable. People with visual impairments need to travel and move, in an independent way through places that often are not prepared for that. A simple task, as for example, go to a doctor's appointment at the hospital, can become transformed in a very difficult task due to the various obstacles that the user must overcome. In last years, several systems were developed that try to address this issue [1][2], some of them are designed for indoor movement, others for outdoor and there are al so systems that consider both scenarios (outdoor and indoor). Despite the existent systems this research area is 
still an open problem, one of the major limitation lies in the fact that the information gathered from the environment is sometimes dotted with uncertainty or incomplete, leading to incorrect decisions. Other issue is the ability to recognize user intentions and therefore provide better assistance.

Decision Support Systems (DSS) are based on a knowledge base (user's characteristics, preferences and goals), with the aim of supporting the user [3]. This type of systems may be completely independent of human interaction and may also take advantage of humans to capture information and process it [3]. DSS may also be framed in ambient intelligence systems. The ambient intelligence (AmI) considers the user at the center of a digital ambient, i.e., AmI is based on an electronic environment that centers the user in a virtual reality that processes information in function of his presence and movements. DSS are sensible and adaptable, being able to meet the user's real needs, habits, attitudes and emotions [4].

The information collected by DSS about the environment will be the basis of all knowledge that will allow supporting users in their decision making processes. However, in a considerable number of situations, the information collected or inferred is incomplete or even uncertain. This may lead to wrong conclusions, which in turn will lead to "wrong support" and "bad decisions". The use of speculative computation to solve problems with missing information or weak conditions of communication has increased in recent years.

In this context, the aim of this work is to propose an architecture able to assist people with visual disabilities, helping those avoiding objects and falls. The ambient information captured by image sensors and devices makes it possible to determine the presence of obstacles (objects, furniture, walls, depth, etc.) in the ambient in which the user is. Through the use of speculative computation, we think it is possible to predict the users' intentions and even to justify the reactive or proactive users' behaviors.

The rest of the paper is organized as follow. First we present the state of the art. Next we present the environment modeling and after it we present a proposed architecture to be applied to a system. In the last section we conclude our work and present the future work.

\section{State of the Art}

Before starting the development process of this project some research of references had to be done in order to support all the project's specifications and all the decisions made. Regarding the research some areas and systems were taken as reference: Ambient Intelligence (Aml), Decision Support Systems (DSS), Speculative Computation and Multi-Agent Systems.

Aml defines a vision of a future in which environments support the people inhabiting them. We can find examples of Aml applications in several 
environments like smart homes, smart offices, intelligent meeting rooms, ambient healthcare, smart classrooms, etc [5][6][7]. However for the purpose of this proposal we will focus on our appliance domain of application, the smart offices and intelligent meeting rooms (IMR) context. The emergence of AmI concept contributes with a new perspective, a different way of viewing traditional offices and decision rooms, where it is expected to include key features like monitoring, prediction, autonomy, adaptation/learning and the ability to naturally communicate with humans. In addition the IMR are also defined as an environment that is able to adapt itself to the user needs by releasing the users from routine tasks they should perform to change the environment to suit to their preferences and to access services available at each moment by customized interfaces [9].

The DSS are computational solutions that might be used to assist on taking complex decisions and problem solving [5]. In this sense there are several systems that integrate decision support who predict the user's intention in order to facilitate his tasks and problems.

In the context of AmI we can find several projects that integrate the recognition of the user's intentions: military projects, used to detect terrorist activity [7] and anticipate the enemy's movements [8] and also to control urban riots [9]. Other projects were ambient support can be found are based on users that need medical care, such as elderly [10] and people with chronic diseases like Alzheimer [11]. Another concept of this type of systems is based on help deficient people like blind people that use a smart helmet with camera sensors for navigation [14].

The speculative computation is a provisional computation that uses propositions by default, i.e., uses assumptions to continue processing, when information is incomplete or even when there is no essential information to gain knowledge [12]. This type of computation was used for the first time in the 80's on parallel processing in order to improve the processing times [13]. At the beginning of the XXI century, the researcher Ken Satoh [12], started applying abductive logic on speculative computation with the objective of processing incomplete information [15][16]. To support these systems, Ken Satoh used a Japanese concept denominated "Kiga-Kiku" so that the system would understand a determined situation and take the most appropriate decision without being explicitly informed of what to do. This concept is applied when an individual is able to predict the others intention's and act proactively in function of preacquired knowledge [17]. Thus this concept, when applied in systems that incorporate speculative computation, aims to understand and know the diverse situations used to generate an answer, for example the perception of the user's intentions by learning what his preferences are. This concept is also able to manipulate incomplete information common to the environments in which the user is inserted [17].

During the past few years new projects to treat incomplete information's in multi-agent systems have appeared. The emergence of these systems is based on incomplete information and problem solving capacities that each agent has. This 
required the development of multi-agent systems with the goal of all working together in order to achieve results in solving problems [18]. This type of system can be applied at a domestic level in order to control the house and distribute tasks.

Overall, we can say that the speculative computation is very useful when we want to process incomplete information. Sometimes, in a decision making context, the decision does not allow a speculative processing. This type of computation can also be used in learning processes in order to generate useful information for future decisions.

\section{Environment Modeling}

The environment modulation is essential to understand the connection between the different models which constitute a particular system. In general, this system should be divided into layers (Figure 1), where each layer processes the information according to its functionality.

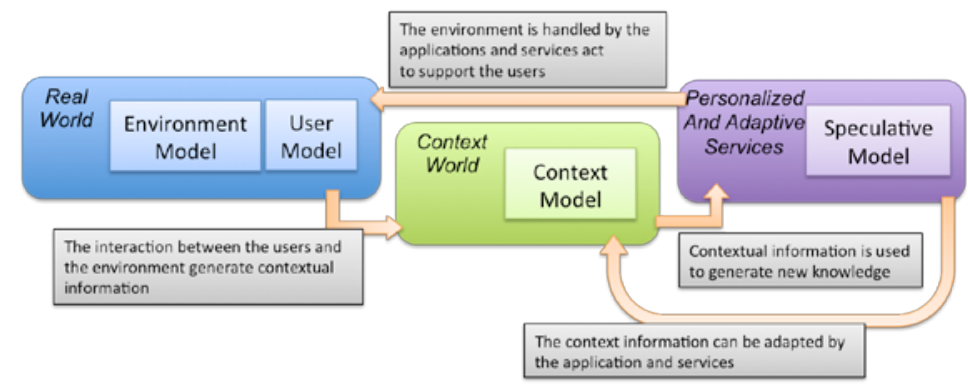

Figure 1. Environment Modeling [adapted by [20]]

The first layer is named Real World and maintains the information acquired by the sensors. Through this model, the information is obtained through image capturing devices, like a video camera, that capture singular or sequence images. The environmental model is the first to interact with the information acquired by the sensors in order to maintain a real representation of the environment in a certain instant of time. The user model maintains specific information of a particular user. This information is very relevant to allow the system to generate the correct answer according to the user needs. Some examples of information that establish the user model are: age, height, weight, deficiencies, etc. Another type of information corresponds to the context in which certain system is operating in order to produce quality results according to the user's goals.

One of the user goals might be, for example, the movement from point $A$ (current location) to a point $B$ (destination). Part of this information is induced through the combination of the information obtained in the first layer, i.e., the 
environment information combined with the user information generates the context information for a certain situation. This information is maintained in the context model, on second layer, Context World.

The third layer, Personalized and Adaptive Services, incorporates the speculative model where all gathered information is interpreted and processed in order to produce answers. The answers that shall produce intend to help the user avoiding obstacles and falls in order to ensure a safe user movement. After the interpretation and processing of the information are produced results that will inform the user, through sonorous commands, about the physical actions that should be taken in order to move, thereby avoiding obstacles and possible falls.

The interconnection of all the layers begins at the first layer, Real World, where all the information is captured in a time instant. Afterwards, assuming that the user model already contains information, the combination of these with the information gathered on the first layer generates context information that is introduced on the second layer, Context World. When all the information is gathered, it's send to the third layer, Per sonalized and Adaptive Services, where, with the help of speculative computation, the parallel processing is performed in order to achieve a solution or a better answer to the problem. The processing result generates new context information that is send to the second layer in order to update the previous information that the system contained. Additionally, this information is send to the first information layer in order to interact with the user aiming to facilitate his movement.

\section{Proposed Architecture}

The definition of the architecture is fundamental because it will facilitate the understanding of the functionalities and the interconnections of all its components. In the environment modulation were identified all the layers and models that will now be integrated on architecture. At this section the goal is to specify the proposed architecture as well as the information flow and processing until the achievement of the results.

As it was previously mentioned, this architecture initiates its function through the acquisition of information by image capture devices. For that, our goal is to use a camera developed by Microsoft Corporation for the use on videogames on the console Xbox. This camera, denominated "Kinect", has the particularity of obtaining a depth matrix from the image captured, i.e., it's possible to know at what distance the objects and/or obstacles are. Through this functionality it is possible to obtain useful information for the following process. In Figure 2 we can have a better perception of the proposed architecture, which starts with the image acquisition and ends at the point that the user is informed of the actions that he need perform. 


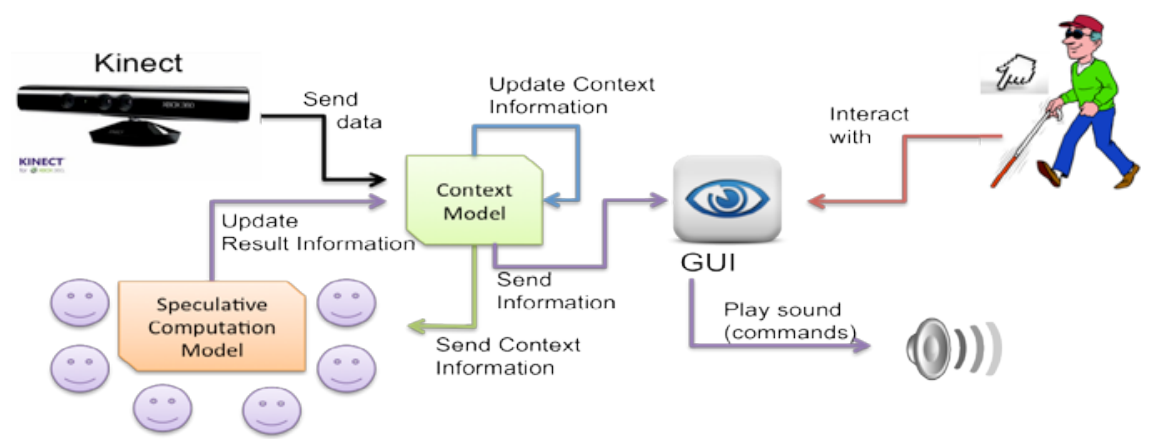

Figure 2. Architecture for support blind people

The aim of this architecture is to predict the human behavior through speculative computation, covering all the possible cases of a determined problem. For this it is intended to include the multi-agents concept, where each agent is responsible of processing part of the information at a certain time instant in order to integrate the function pattern of the speculative computation and also reduce the processing load.

For a better understanding of the speculative module model function it will be explained how the information is processed. The information processing starts with the capture of information, through the camera, and ends with its processing by the speculative module model in order to obtain answers according to the user's needs. When the information is transmitted to each system agent, the process is initialized in order to generate a logic tree until a response is obtained from the corresponding scenario (Figure 3).

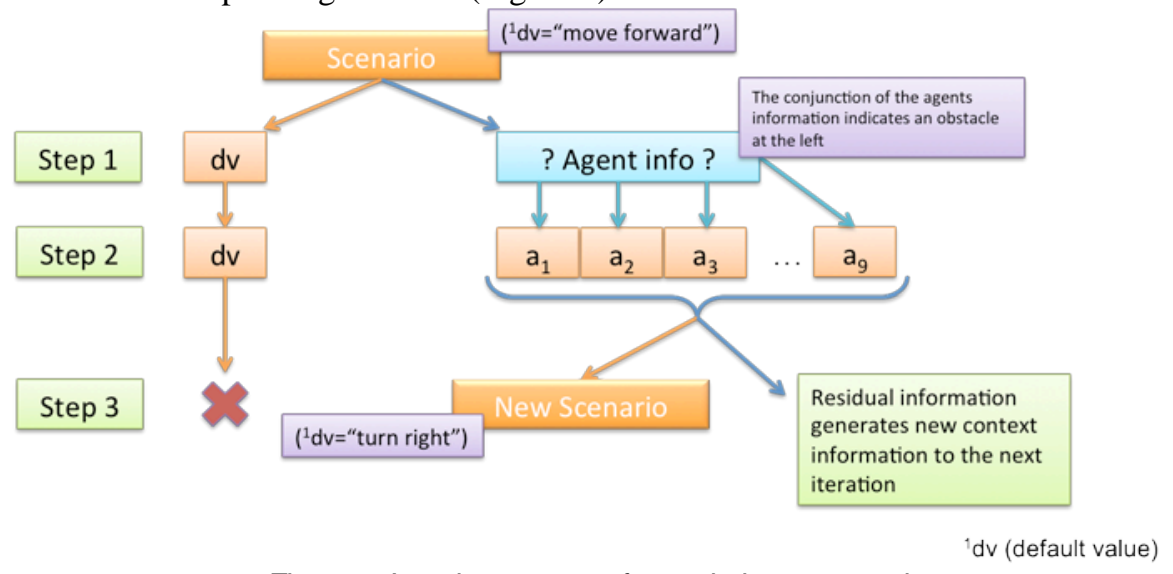

Figure 3. Iteration process of speculative computation

The possible scenarios for this system might be: move forward, turn or deviate to the left or to the right and depth detected. 
At the bottom of the tree, generated during the processing, is instantiated a value (e.g. "move forward") that might be changed during the tree construction when any contradiction is assumed. A contradiction is assumed when the default value (e.g. "move forward") has to be changed to another value due to the combination of information from the agents. When the information received by the agents does not contradict the initial value of the tree this value is maintained until the next iteration. Thus, the initial value is considered viable and truth until some agent contradicts it. At the end of an iteration the information transmitted by the agents, even if it doesn't contradict the initial value, is used to generate new context information for the next iteration.

\section{Conclusions and Future Work}

In this paper we propose a context aware architecture to support blind people. This architecture was made to be inserted in a multi-agent system. The focus of this architecture is support the blind people to execute some tasks in ambient intelligence context. This process collects information about the ambient intelligence by a sensorial device and the next step is to convert the information in knowledge to support the user decision making.

Our aim to the future work is to develop a multi-agent system based in the proposed architecture to be applied in an ambient intelligence context. The aim is to support the blind people to make better decisions.

\section{Acknowledgments}

This work is part-funded by ERDF - European Regional Development Funds through the COMPETE Programme (operational programme for competitiveness) and by National Funds through the FCT - Fundação para a Ciéncia e a Tecnologia (Portuguese Foundation for Science and Technology) within project FCOMP-01-0124-FEDER-028980 (PTDC/EEI-SII/1386/2012).

\section{References}

[1] Pinedo, M., Villanueva, F., Santofimia M., López J. (2011) Multimodal Positioning Support for Ambient Intelligence. 5th International Symposium on Ubiquitous Computing and Ambient Intelligence, pp. 1-8.

[2] Guerrero, L., Vasquez, F. and Ochoa, S. (2012) An Indoor Navigation System for the Visually Impaired, Sensors (Basel). vol. 12, no 6, pp. 8236-8258. 
[3] Shim, J., Warkentin, M., Courtney, J., Power, D., Sharda, R., Carlsson, C. (2002) Past, present, and future of decision support technology. Decision Support Systems, vol. 33, no. 2, pp. 111-126.

[4] Preuveneers, D. and Novais, P. (2012) A survey of software engineering best practices for the development of smart applications in Ambient Intelligence. Journal of Ambient Intelligence and Smart Environments, vol. 4, no. 3, pp. 149-162.

[5] Sadri, F. (2011). Ambient intelligence: A survey. ACM Computing Surveys (CSUR). vol. 43 issue 4, no. 36, pp. 36-66, New York, USA.

[6] Ramos, C., Augusto, J., Shapiro, D. (2008) Ambient Intelligence- - the Next Step for Artificial Intelligence. Intelligent Systems, IEEE, vol. 23, pp. 15-18.

[7] Cook, D. Augusto, J., Jakkula, V. (2009) Ambient Intelligence: applications in society and opportunities for AI. Pervasive and Mobile Computing. vol. 5, no.4, pp. 277- 298. Elsevier.

[8] Mao, W., Gratch, J. (2004) A utility-based approach to intention recognition. AAMAS 2004 Workshop on Agent Tracking: Modelling Other Agents from Observations.

[9] Suzic, R., Svenson, P., (2006) Capabilities-based plan recognition. 9th International Conference on Information Fusion, pp.1-7, Florence, Italy.

[10] Pereira, L. and Anh, H. (2009) Elder care via intention recognition and evolution prospection. 18th International Conference on Applications of Declarative Programming and Knowledge Managment (INAP'09), Évora, Portugal.

[11] Roy, P., Bouchard, B., Bouzouane, A., Giroux, S. (2007) A hybrid plan recognition model for Alzheimer's patients: interleaved-erroneous dilemma. IEEE/WIC/ACM International Conference on Intelligent Agent Technology, pp. 131-137, California, USA.

[12] Satoh, K., Inoue, K., Iwanuma, K., and Sakama, C. (2000) Speculative Computation by Abduction under Incomplete Communication Environments, ICMAS2000, pp. 263-270.

[13] Burton, F. (1985) Speculative Computation, Parallelism and Functional Programming. IEEE Transactions on Computers, vol. c-34, pp.1190-1193.

[14] Mann,S., Huang, J., Janzen, R., Lo, R., Rampersad, V., Chen, A., Doha, T. (2011) Blind navigation with a wearable range camera and vibrotactile helmet. Proceedings of the 19th ACM international conference on Multimedia - MM '11, p. 1325, USA.

[15] Satoh, K. and Yamamoto, K. (2002). Speculative computation with multi-agent belief revision. International Joint Conference on Autonomous Agents and Multiagent Systems (AAMAS), pp. 897-904, New York, USA. ACM Press.

[16] Satoh, K. (2005) Speculative computation and abduction for an autonomous agent, IEICE Transactions, vol. 88-D, no. 9, pp. 2031-2038.

[17] Satoh, K., Kiga-kiku computing and speculative computation (2012), Awareness: Self-Awareness in Automic Systems.

[18] Sycara, K. (1998) Multiagent Systems, AI Magazine, vol. 19, no. 2, pp. 79-92.

[19] Garcia-Valverde, T., Serrano, E., Botia, J. (2010) Combining the real world with simulations for a robust testing of ambient intelligence services. Artificial Intelligence Review, pp. 1-24. 